\title{
On the convergence of high-order Ehrlich-type iterative methods for approximating all zeros of a polynomial simultaneously
}

\author{
Petko D Proinov ${ }^{*}$ and Maria T Vasileva
}

\section{"Correspondence:}

proinov@uni-plovdiv.bg

Faculty of Mathematics and

Informatics, University of Plovdiv

Plovdiv, 4000, Bulgaria

\begin{abstract}
We study a family of high-order Ehrlich-type methods for approximating all zeros of a polynomial simultaneously. Let us denote by $T^{(1)}$ the famous Ehrlich method (1967). Starting from $T^{(1)}$, Kjurkchiev and Andreev (1987) have introduced recursively a sequence $\left(T^{(N)}\right)_{N=1}^{\infty}$ of iterative methods for simultaneous finding polynomial zeros. For given $N \geq 1$, the Ehrlich-type method $T^{(N)}$ has the order of convergence $2 N+1$. In this paper, we establish two new local convergence theorems as well as a semilocal convergence theorem (under computationally verifiable initial conditions and with an a posteriori error estimate) for the Ehrlich-type methods $T^{(\mathrm{M})}$. Our first local convergence theorem generalizes a result of Proinov (2015) and improves the result of Kjurkchiev and Andreev (1987). The second local convergence theorem generalizes another recent result of Proinov (2015), but only in the case of the maximum norm. Our semilocal convergence theorem is the first result in this direction.
\end{abstract}

MSC: Primary 65H04; secondary $12 \mathrm{Y} 05$

Keywords: simultaneous methods; Ehrlich method; polynomial zeros; accelerated convergence; local convergence; semilocal convergence; error estimates

\section{Introduction}

Throughout this paper $(\mathbb{K},|\cdot|)$ denotes an algebraically closed field and $\mathbb{K}[z]$ denotes the ring of polynomials (in one variable) over $\mathbb{K}$. For a given vector $x$ in $\mathbb{K}^{n}, x_{i}$ always denotes the $i$ th coordinate of $x$. In particular, if $F$ is a map with values in $\mathbb{K}^{n}$, then $F_{i}(x)$ denotes the $i$ th coordinate of the vector $F(x)$. We endow the vector space $\mathbb{K}^{n}$ with the norm $\|x\|_{p}$ defined as usual:

$$
\|x\|_{p}=\left(\sum_{i=1}^{n}\left|x_{i}\right|^{p}\right)^{1 / p} \quad \text { for } 1 \leq p<\infty ; \quad\|x\|_{\infty}=\max \left\{\left|x_{1}\right|, \ldots,\left|x_{n}\right|\right\} .
$$

We endow $\mathbb{R}^{n}$ with the coordinate-wise ordering $\preceq$ defined by

$$
x \preceq y \quad \text { if and only if } \quad x_{i} \leq y_{i} \text { for each } i=1, \ldots, n .
$$

(c) 2015 Proinov and Vasileva. This article is distributed under the terms of the Creative Commons Attribution 4.0 International License (http://creativecommons.org/licenses/by/4.0/), which permits unrestricted use, distribution, and reproduction in any medium, provided you give appropriate credit to the original author(s) and the source, provide a link to the Creative Commons license, and indicate if changes were made. 
Then $\left(\mathbb{R}^{n},\|\cdot\|_{p}\right)$ is a solid vector space. We endow $\mathbb{K}^{n}$ with the cone norm $\|\cdot\|: \mathbb{K}^{n} \rightarrow \mathbb{R}^{n}$ defined by

$$
\|x\|=\left(\left|x_{1}\right|, \ldots,\left|x_{n}\right|\right) \text {. }
$$

Then $\left(\mathbb{K}^{n},\|\cdot\|, \preceq\right.$ ) is a cone normed space over $\mathbb{R}^{n}$ (see, e.g., Proinov [1]).

Let $f \in \mathbb{K}[z]$ be a polynomial of degree $n \geq 2$. A vector $\xi \in \mathbb{K}^{n}$ is said to be a root vector of $f$ if

$$
f(z)=a_{0} \prod_{i=1}^{n}\left(z-\xi_{i}\right) \quad \text { for all } z \in \mathbb{K},
$$

where $a_{0} \in \mathbb{K}$. Obviously, $f$ has a root vector in $\mathbb{K}^{n}$ if and only if it splits in $\mathbb{K}$. We denote by $\operatorname{sep}(f)$ the separation number of $f$ which is defined to be the minimum distance between two distinct zeros of $f$, that is,

$$
\operatorname{sep}(f)=\min \{|\xi-\eta|: f(\xi)=f(\eta), \xi \neq \eta\}
$$

\subsection{The Weierstrass method and Weierstrass correction}

In the literature, there are a lot of iterative methods for finding all zeros of $f$ simultaneously (see, e.g., the monographs of Sendov et al. [2], Kyurkchiev [3], McNamee [4] and Petković [5] and references given therein). In 1891, Weierstrass [6] published his famous iterative method for simultaneous computation of all zeros of $f$. The Weierstrass method is defined by the following iteration:

$$
x^{(k+1)}=x^{(k)}-W_{f}\left(x^{(k)}\right), \quad k=0,1,2, \ldots,
$$

where the operator $W_{f}: \mathcal{D} \subset \mathbb{K}^{n} \rightarrow \mathbb{K}^{n}$ is defined by

$$
W_{f}(x)=\left(W_{1}(x), \ldots, W_{n}(x)\right) \quad \text { with } \quad W_{i}(x)=\frac{f\left(x_{i}\right)}{a_{0} \prod_{j \neq i}\left(x_{i}-x_{j}\right)} \quad(i=1, \ldots, n),
$$

where $a_{0} \in \mathbb{K}$ is the leading coefficient of $f$ and the domain $\mathcal{D}$ of $W$ is the set of all vectors in $\mathbb{K}^{n}$ with distinct components. The Weierstrass method (1.5) has second order of convergence provided that all zeros of $f$ are simple. The operator $W_{f}$ is called Weierstrass correction. We should note that $W_{f}$ plays an important role in many semilocal convergence theorems for simultaneous methods.

\subsection{The Ehrlich method}

Another famous iterative method for finding simultaneously all zeros of a polynomial $f$ was introduced by Ehrlich [7] in 1967. The Ehrlich method is defined by the following fixed point iteration:

$$
x^{(k+1)}=T\left(x^{(k)}\right), \quad k=0,1,2, \ldots,
$$

where the operator $T: \mathscr{D} \subset \mathbb{K}^{n} \rightarrow \mathbb{K}^{n}$ is defined by $T(x)=\left(T_{1}(x), \ldots, T_{n}(x)\right)$ with

$$
T_{i}(x)=x_{i}-\frac{f\left(x_{i}\right)}{f^{\prime}\left(x_{i}\right)-f\left(x_{i}\right) \sum_{j \neq i} \frac{1}{x_{i}-x_{j}}} \quad(i=1, \ldots, n)
$$


and the domain of $T$ is the set

$$
\mathscr{D}=\left\{x \in \mathcal{D}: f^{\prime}\left(x_{i}\right)-f\left(x_{i}\right) \sum_{j \neq i} \frac{1}{x_{i}-x_{j}} \neq 0 \text { for } i \in I_{n}\right\} .
$$

Here and throughout the paper, we denote by $I_{n}$ the set of indices $1, \ldots, n$, that is, $I_{n}=\{1, \ldots, n\}$. The Ehrlich method has third order of convergence if all zeros of $f$ are simple. The Ehrlich method was rediscovered by Abert [8] in 1973. In 1970, Börsch-Supan [9] introduced another third-order method for numerical computation of all zeros of a polynomial simultaneously. In 1982, Werner [10] has proved that the both methods are identical. The Ehrlich method (1.7) is known also as 'Ehrlich-Abert method', 'Börsch-Supan method', and 'Abert method'.

Recently, Proinov [11] obtained two local convergence theorems for Ehrlich method under different types of initial conditions. The first one generalizes and improves the results of Kyurkchiev and Tashev [12,13] and Wang and Zhao [14], Theorem 2.1. The second one generalizes and improves the results of Wang and Zhao [14], Theorem 2.2 and Tilli [15], Theorem 3.3.

Before we state the two results of [11], we need some notations which will be used throughout the paper. For given vectors $x \in \mathbb{K}^{n}$ and $y \in \mathbb{R}^{n}$, we define in $\mathbb{R}^{n}$ the vector

$$
\frac{x}{y}=\left(\frac{\left|x_{1}\right|}{y_{1}}, \ldots, \frac{\left|x_{n}\right|}{y_{n}}\right)
$$

provided that $y$ has no zero components. Given $p$ such that $1 \leq p \leq \infty$, we always denote by $q$ the conjugate exponent of $p$, i.e. $q$ is defined by means of

$$
1 \leq q \leq \infty \quad \text { and } \quad 1 / p+1 / q=1
$$

In the sequel, we use the function $d: \mathbb{K}^{n} \rightarrow \mathbb{R}^{n}$ defined by $d(x)=\left(d_{1}(x), \ldots, d_{n}(x)\right)$ with

$$
d_{i}(x)=\min _{j \neq i}\left|x_{i}-x_{j}\right| \quad(i=1, \ldots, n)
$$

Let $a>0$ and $b \geq 1$. We define the real function $\phi$ by

$$
\phi(t)=\frac{a t^{2}}{(1-t)(1-b t)-a t^{2}}
$$

and the real number $R$ as follows:

$$
R=\frac{2}{b+1+\sqrt{(b-1)^{2}+8 a}} \text {. }
$$

Theorem 1.1 (Proinov [11]) Let $f \in \mathbb{K}[z]$ be a polynomial of degree $n \geq 2$ which has only simple zeros, $\xi \in \mathbb{K}^{n}$ be a root vector of $f$ and $1 \leq p \leq \infty$. Suppose $x^{(0)} \in \mathbb{K}^{n}$ is an initial guess satisfying

$$
E\left(x^{(0)}\right)=\left\|\frac{x^{(0)}-\xi}{d(\xi)}\right\|_{p}<R=\frac{2}{b+1+\sqrt{(b-1)^{2}+8 a}},
$$


where $a=(n-1)^{1 / q}$ and $b=2^{1 / q}$. Then the Ehrlich iteration (1.7) is well defined and converges cubically to $\xi$ with error estimates

$$
\left\|x^{(k+1)}-\xi\right\| \preceq \lambda^{3^{k}}\left\|x^{(k)}-\xi\right\| \quad \text { and } \quad\left\|x^{(k)}-\xi\right\| \preceq \lambda^{\left(3^{k}-1\right) / 2}\left\|x^{(0)}-\xi\right\|
$$

for all $k \geq 0$, where $\lambda=\phi\left(E\left(x^{(0)}\right)\right)$ and the function $\phi$ is defined by (1.10).

Theorem 1.2 (Proinov [11]) Let $f \in \mathbb{K}[z]$ be a polynomial of degree $n \geq 2, \xi \in \mathbb{K}^{n}$ be a root vector off and $1 \leq p \leq \infty$. Suppose $x^{(0)} \in \mathbb{K}^{n}$ is a vector with distinct components satisfying

$$
E\left(x^{(0)}\right)=\left\|\frac{x^{(0)}-\xi}{d\left(x^{(0)}\right)}\right\|_{p} \leq R=\frac{2}{b+1+\sqrt{(b-1)^{2}+8 a}}
$$

where $a=(n-1)^{1 / q}$ and $b=2^{1 / q}$. Then $f$ has only simple zeros in $\mathbb{K}$ and Ehrlich iteration (1.7) is well defined and converges to $\xi$ with error estimates

$$
\left\|x^{(k+1)}-\xi\right\| \preceq \theta \lambda^{3^{k}}\left\|x^{(k)}-\xi\right\| \quad \text { and } \quad\left\|x^{(k)}-\xi\right\| \preceq \theta^{k} \lambda^{\left(3^{k}-1\right) / 2}\left\|x^{(0)}-\xi\right\|
$$

for all $k \geq 0$, where $\lambda=\phi\left(E\left(x^{(0)}\right)\right), \theta=\psi\left(E\left(x^{(0)}\right)\right)$ and the function $\phi$ is defined by (1.10) and the function $\psi$ by

$$
\psi(t)=\frac{(1-t)(1-b t)-a t^{2}}{1-t-a t^{2}} .
$$

Moreover, the method converges cubically to $\xi$ provided that $E\left(x^{(0)}\right)<R$.

\subsection{A family of high-order Ehrlich-type methods}

In the following definition, we define a sequence $\left(T^{(N)}\right)_{N=0}^{\infty}$ of iteration functions in the vector space $\mathbb{K}^{n}$. In what follows, we define the binary relation \# on $\mathbb{K}^{n}$ by

$$
x \# y \quad \Leftrightarrow \quad x_{i} \neq y_{j} \quad \text { for all } i, j \in I_{n} \text { with } i \neq j .
$$

Definition 1.3 Let $f \in \mathbb{K}[z]$ be a polynomial of degree $n \geq 2$. Define the sequence $\left(T^{(N)}\right)_{N=0}^{\infty}$ of functions $T^{(N)}: D_{N} \subset \mathbb{K}^{n} \rightarrow \mathbb{K}^{n}$ recursively by setting $T^{(0)}(x)=x$ and

$$
T_{i}^{(N+1)}(x)=x_{i}-\frac{f\left(x_{i}\right)}{f^{\prime}\left(x_{i}\right)-f\left(x_{i}\right) \sum_{j \neq i} \frac{1}{x_{i}-T_{j}^{(N)}(x)}} \quad(i=1, \ldots, n),
$$

where the sequence of domains $\left(D_{N}\right)_{N=0}^{\infty}$ is also defined recursively by setting $D_{0}=\mathbb{K}^{n}$ and

$$
D_{N+1}=\left\{x \in D_{N}: x \# T^{(N)}(x), f^{\prime}\left(x_{i}\right)-f\left(x_{i}\right) \sum_{j \neq i} \frac{1}{x_{i}-T_{j}^{(N)}(x)} \neq 0 \text { for } i \in I_{n}\right\} .
$$

Given $N \in \mathbb{N}$, the $N$ th method of Kjurkchiev-Andreev's family can be defined by the following fixed point iteration:

$$
x^{(k+1)}=T^{(N)}\left(x^{(k)}\right), \quad k=0,1,2, \ldots
$$


It is easy to see that in the case $N=1$ the Ehrlich-type method (1.18) coincides with the classical Ehrlich method (1.7). The order of convergence of the Ehrlich-type method (1.18) is $2 N+1$.

Kjurkchiev and Andreev [16] established the following convergence result for the Ehrlich-type methods (1.18). This result and its proof can also be found in the monographs of Sendov, Andreev and Kjurkchiev [2], Section 19 and Kyurkchiev [3], Chapter 9.2).

Theorem 1.4 (Kjurkchiev and Andreev [16]) Let $f \in \mathbb{C}[z]$ be a polynomial of degree $n \geq 2$ which has only simple zeros, $\xi \in \mathbb{C}^{n}$ be a root vector off and $N \geq 1$. Let $0<h<1$ and $c>0$ be such that

$$
\delta>2 c(1+(2 n-1) h) \quad \text { and } \quad \frac{n c^{2}}{(\delta-c)(\delta-2 c-2 c h)-3(n-1) c^{2} h^{2}} \leq 1,
$$

where $\delta=\operatorname{sep}(f)$ defined by (1.4). Suppose $x^{(0)} \in \mathbb{C}^{n}$ is an initial guess satisfying the condition

$$
\left\|x^{(0)}-\xi\right\|_{\infty} \leq c h
$$

Then the Ehrlich-type method (1.18) converges to $\xi$ with error estimate

$$
\left\|x^{(k)}-\xi\right\|_{\infty} \leq \operatorname{ch}^{(2 N+1)^{k}} \quad \text { for all } k \geq 0
$$

\subsection{The purpose of the paper}

In this paper, we present two new local convergence theorems as well as a semilocal convergence theorem (under computationally verifiable initial conditions and with an $a$ posteriori error estimate) for Ehrlich-type methods (1.18). Our first local convergence result (Theorem 4.6) generalizes Theorem 1.1 (Proinov [11]) and improves Theorem 1.4 (Kjurkchiev and Andreev [16]). Our second local convergence result (Theorem 5.4) generalizes Theorem 1.2 (Proinov [11]), but only in the case $p=\infty$. Furthermore, several numerical examples are provided to show some practical applications of our semilocal convergence result.

\section{A general convergence theorem}

Recently, Proinov [17-19] has developed a general convergence theory for iterative processes of the type

$$
x_{k+1}=T x_{k}, \quad k=0,1,2, \ldots
$$

where $T: D \subset X \rightarrow X$ is an iteration function in a cone metric space $X$. In order to make this paper self-contained, we briefly review some basic definitions and results from this theory.

Throughout this paper $J$ denotes an interval on $\mathbb{R}_{+}$containing 0 . For an integer $k \geq 1$, we denote by $S_{k}(t)$ the following polynomial:

$$
S_{k}(t)=1+t+\cdots+t^{k-1}
$$

If $k=0$ we assume that $S_{k}(t) \equiv 0$. Throughout the paper we assume by definition that $0^{0}=1$. 
Definition 2.1 ([18]) A function $\varphi: J \rightarrow \mathbb{R}_{+}$is called quasi-homogeneous of degree $r \geq 0$ on $J$ if it satisfies the following condition:

$$
\varphi(\lambda t) \leq \lambda^{r} \varphi(t) \quad \text { for all } \lambda \in[0,1] \text { and } t \in J \text {. }
$$

If $m$ functions $\varphi_{1}, \ldots, \varphi_{m}$ are quasi-homogeneous on $J$ of degree $r_{1}, \ldots, r_{m}$, then their product $\varphi_{1} \cdots \varphi_{m}$ is a quasi-homogeneous function of degree $r_{1}+\cdots+r_{m}$ on $J$. Note also that a function $\varphi$ is quasi-homogeneous of degree 0 on $J$ if and only it is nondecreasing on $J$.

Definition 2.2 ([17]) A function $\varphi: J \rightarrow J$ is said to be a gauge function of order $r \geq 1$ on $J$ if it satisfies the following conditions:

(i) $\varphi$ is quasi-homogeneous of degree $r$ on $J$;

(ii) $\varphi(t) \leq t$ for all $t \in J$.

A gauge function $\varphi$ of order $r$ on $J$ is said to be a strict gauge function if the inequality in (ii) holds strictly whenever $t \in J \backslash\{0\}$.

The following is a sufficient condition for a gauge function of order $r$.

Lemma 2.3 ([18]) If $\varphi: J \rightarrow \mathbb{R}_{+}$is a quasi-homogeneous function of degree $r \geq 1$ on an interval $J$ and $R>0$ is a fixed point of $\varphi$ in $J$, then $\varphi$ is a gauge function of order $r$ on $[0, R]$. Moreover, if $r>1$, then function $\varphi$ is a strict gauge of order $r$ on $J=[0, R)$.

Definition 2.4 ([17]) Let $T: D \subset X \rightarrow X$ be a map on an arbitrary set $X$. A function $E: D \rightarrow \mathbb{R}_{+}$is said to be a function of the initial conditions of $T$ (with a gauge function $\varphi$ on $J$ ) if there exists a function $\varphi: J \rightarrow J$ such that

$$
E(T x) \leq \varphi(E(x)) \quad \text { for all } x \in D \text { with } T x \in D \text { and } E(x) \in J
$$

Definition 2.5 ([17]) Let $T: D \subset X \rightarrow X$ be a map on an arbitrary set $X$, and $E: D \rightarrow \mathbb{R}_{+}$ be a function of the initial conditions of $T$ with a gauge function on $J$. Then a point $x \in D$ is said to be an initial point of $T$ (with respect to $E$ ) if $E(x) \in J$ and all of the iterates $T^{k} x$ $(k=0,1,2, \ldots)$ are well defined and belong to $D$.

The following is a simple sufficient condition for initial points.

Theorem 2.6 ([18]) Let $T: D \subset X \rightarrow X$ be a map on an arbitrary set $X$ and $E: D \rightarrow \mathbb{R}_{+}$ be a function of the initial conditions of $T$ with a gauge function $\varphi$ on J. Suppose that $x \in D$ with $E(x) \in J$ implies $T x \in D$. Then every point $x_{0} \in D$ such that $E\left(x_{0}\right) \in J$ is an initial points of $T$.

Definition 2.7 ([19]) Let $T: D \subset X \rightarrow X$ be an operator in a cone normed space $(X,\|\cdot\|)$ over a solid vector space $(Y, \preceq)$, and let $E: D \rightarrow \mathbb{R}_{+}$be a function of the initial conditions of $T$ with a gauge function on an interval $J$. Then the operator $T$ is said to be an iterated contraction at a point $\xi \in D$ (with respect to $E$ ) if $E(\xi) \in J$ and

$$
\|T x-\xi\| \preceq \beta(E(x))\|x-\xi\| \quad \text { for all } x \in D \text { with } E(x) \in J,
$$

where the control function $\beta: J \rightarrow[0,1)$ is nondecreasing. 
The following fixed point theorem plays an important role in our paper.

Theorem 2.8 (Proinov [19]) Let $T: D \subset X \rightarrow X$ be an operator of a cone normed space $(X,\|\cdot\|)$ over a solid vector space $(Y, \preceq)$, and let $E: D \rightarrow \mathbb{R}_{+}$be a function of the initial conditions of $T$ with a gauge function $\varphi$ of order $r \geq 1$ on an interval J. Suppose $T$ is an iterated contraction at a point $\xi$ with respect to $E$ with control function $\beta$ such that

$$
t \beta(t) \text { is a strict gauge function of order } r \text { on } J
$$

and there exists a function $\psi: J \rightarrow \mathbb{R}_{+}$such that

$$
\beta(t)=\phi(t) \psi(t) \quad \text { for all } t \in J
$$

where $\phi: J \rightarrow \mathbb{R}_{+}$is a nondecreasing function satisfying

$$
\varphi(t)=t \phi(t) \quad \text { for all } t \in J .
$$

Then the following statements hold true.

(i) The point $\xi$ is a unique fixed point of $T$ in the set $U=\{x \in D: E(x) \in J\}$.

(ii) Starting from each initial point $x^{(0)}$ of $T$, Picard iteration (2.1) remains in the set $U$ and converges to $\xi$ with error estimates

$$
\left\|x^{(k+1)}-\xi\right\| \preceq \theta \lambda^{r^{k}}\left\|x^{(k)}-\xi\right\| \text { and }\left\|x^{(k)}-\xi\right\| \preceq \theta^{k} \lambda^{S_{k}(r)}\left\|x^{(0)}-\xi\right\|
$$

for all $k \geq 0$, where $\lambda=\phi\left(E\left(x^{(0)}\right)\right)$ and $\theta=\psi\left(E\left(x^{(0)}\right)\right)$.

In the case $\beta \equiv \phi$, Theorem 2.8 reduces to the following result.

Corollary 2.9 ([19]) Let $T: D \subset X \rightarrow X$ be an operator in a cone normed space $(X,\|\cdot\|)$ over a solid vector space $(Y, \preceq)$, and let $E: D \rightarrow \mathbb{R}_{+}$be a function of the initial conditions of $T$ with a strict gauge function $\varphi$ of order $r \geq 1$ on an interval J. Suppose that $T$ is an iterated contraction at a point $\xi$ with respect to $E$ and with control function $\phi$ satisfying (2.7). Then the following statements hold true.

(i) The point $\xi$ is a unique fixed point of $T$ in the set $U=\{x \in D: E(x) \in J\}$.

(ii) Starting from each initial point $x^{(0)}$ of $T$, Picard iteration (2.1) remains in $U$ and converges to $\xi$ with order $r$ and error estimates

$$
\left\|x^{(k+1)}-\xi\right\| \preceq \lambda^{r^{k}}\left\|x^{(k)}-\xi\right\| \text { and }\left\|x^{(k)}-\xi\right\| \preceq \lambda^{S_{k}(r)}\left\|x^{(0)}-\xi\right\|
$$

for all $k \geq 0$, where $\lambda=\phi\left(E\left(x^{(0)}\right)\right)$.

\section{Some inequalities in $\mathbb{K}^{n}$}

In this section, we present some useful inequalities in $\mathbb{K}^{n}$ which play an important role in the paper.

Lemma 3.1 ([20]) Let $u, v \in \mathbb{K}^{n}, v$ be a vector with distinct components and $1 \leq p \leq \infty$. Then for all $i, j \in I_{n}$,

$$
\left|u_{i}-v_{j}\right| \geq\left(1-\left\|\frac{u-v}{d(v)}\right\|_{p}\right)\left|v_{i}-v_{j}\right|
$$




$$
\left|u_{i}-u_{j}\right| \geq\left(1-2^{1 / q}\left\|\frac{u-v}{d(v)}\right\|_{p}\right)\left|v_{i}-v_{j}\right| .
$$

Lemma $3.2([19])$ Let $u, v \in \mathbb{K}^{n}$ and $1 \leq p \leq \infty$. If the vector $v$ has distinct components and

$$
\left\|\frac{u-v}{d(v)}\right\|_{p}<\frac{1}{2}
$$

then the vector $u$ also has distinct components.

Lemma 3.3 ([21]) Let $u, v, \xi \in \mathbb{K}^{n}$, $\xi$ be a vector with distinct components, $1 \leq p \leq \infty$ and

$$
\|v-\xi\| \preceq\|u-\xi\|
$$

Then for all $i, j \in I_{n}$,

$$
\left|u_{i}-v_{j}\right| \geq\left(1-2^{1 / q}\left\|\frac{u-\xi}{d(\xi)}\right\|_{p}\right)\left|\xi_{i}-\xi_{j}\right| .
$$

Lemma 3.4 Let $u, v, \xi \in \mathbb{K}^{n}, \alpha \geq 0$, and $1 \leq p \leq \infty$. If $v$ is a vector with distinct components such that

$$
\|u-\xi\| \preceq \alpha\|v-\xi\|,
$$

then for all $i, j \in I_{n}$,

$$
\left|u_{j}-v_{i}\right| \geq\left(1-(1+\alpha)\left\|\frac{v-\xi}{d(v)}\right\|_{p}\right)\left|v_{i}-v_{j}\right| .
$$

Proof By the triangle inequality of cone norm in $\mathbb{K}^{n}$ and (3.5), we obtain

$$
\|u-v\| \preceq\|u-\xi\|+\|v-\xi\| \preceq(1+\alpha)\|v-\xi\|,
$$

which yields

$$
\left\|\frac{u-v}{d(v)}\right\| \preceq(1+\alpha)\left\|\frac{v-\xi}{d(v)}\right\| .
$$

Taking the $p$-norm, we get

$$
\left\|\frac{u-v}{d(v)}\right\|_{p} \leq(1+\alpha)\left\|\frac{v-\xi}{d(v)}\right\|_{p} .
$$

From (3.1) and (3.7), we obtain (3.6), which completes the proof.

Lemma 3.5 Let $u, v, \xi \in \mathbb{K}^{n}, \alpha \geq 0$, and $1 \leq p \leq \infty$. If $v$ is a vector with distinct components such that (3.5) holds, then for all $i, j \in I_{n}$,

$$
\left|u_{i}-u_{j}\right| \geq\left(1-2^{1 / q}(1+\alpha)\left\|\frac{v-\xi}{d(v)}\right\|_{p}\right)\left|v_{i}-v_{j}\right| .
$$

Proof From (3.2) and (3.7), we get (3.8), which completes the proof. 


\section{Local convergence theorem of the first type}

Let $f \in \mathbb{K}[z]$ be a polynomial of degree $n \geq 2$ which has only simple zeros in $\mathbb{K}$, and let $\xi \in \mathbb{K}^{n}$ be a root vector of $f$. In this section we study the convergence of the Ehrlich-type methods (1.18) with respect to the function of the initial conditions $E: \mathbb{K}^{n} \rightarrow \mathbb{R}_{+}$defined as follows:

$$
E(x)=\left\|\frac{x-\xi}{d(\xi)}\right\|_{p} \quad(1 \leq p \leq \infty) .
$$

Let $a>0$ and $b \geq 1$. Throughout this section, we define the function $\phi$ and the real number $R$ by (1.10) and (1.11), respectively. It is easy to show that $R$ is the smallest positive solution of the equation $\phi(t)=1$. Note that $\phi$ is an increasing function which maps $[0, R]$ onto $[0,1]$. Besides, $\phi$ is quasi-homogeneous of degree 2 on $[0, R]$. In the next definition, we introduce a sequence of such functions.

Definition 4.1 We define the sequence $\left(\phi_{N}\right)_{N=0}^{\infty}$ of nondecreasing functions $\phi_{N}:[0, R] \rightarrow[0,1]$ recursively by setting $\phi_{0}(t)=1$ and

$$
\phi_{N+1}(t)=\frac{a t^{2} \phi_{N}(t)}{(1-t)(1-b t)-a t^{2} \phi_{N}(t)},
$$

where $a>0$ and $b \geq 1$ are constants.

Proof of the correctness of Definition 4.1 We prove the correctness of the definition by induction. For $N=0$ it is obvious. Assume that for some $N \geq 0$ the function $\phi_{N}$ is well defined and nondecreasing on $[0, R]$ and $\phi_{N}(R)=1$. We shall prove the same for $\phi_{N+1}$. It follows from the induction hypothesis that

$$
(1-t)(1-b t)-a t^{2} \phi_{N}(t) \geq(1-t)(1-b t)-a t^{2}>0 \quad \text { for all } t \in[0, R]
$$

which means that the function $\phi_{N+1}$ is well defined on $[0, R]$. From (4.2) and the induction hypothesis, we deduce that $\phi_{N+1}$ is nondecreasing on $[0, R]$. From $(4.2)$ and $\phi_{N}(R)=1$, we obtain

$$
\phi_{N+1}(R)=\frac{a R^{2} \phi_{N}(R)}{(1-R)(1-b R)-a R^{2} \phi_{N}(R)}=\frac{a R^{2}}{(1-R)(1-b R)-a R^{2}}=\phi(R)=1 .
$$

This completes the induction and the proof of the correctness of Definition 4.1.

Definition 4.2 For any integer $N \geq 0$, we define the function $\varphi_{N}:[0, R] \rightarrow[0, R]$ as follows:

$$
\varphi_{N}(t)=t \phi_{N}(t)
$$

where the function $\phi_{N}$ is defined by Definition 4.1.

In the next lemma, we present some properties of the functions $\phi_{N}$ and $\varphi_{N}$.

Lemma 4.3 Let $N \geq 0$. Then: 
(i) $\phi_{N}$ is a quasi-homogeneous function of degree $2 N$ on $[0, R]$;

(ii) $\phi_{N+1}(t) \leq \phi(t) \phi_{N}(t)$ for every $t \in[0, R]$;

(iii) $\phi_{N+1}(t) \leq \phi_{N}(t)$ for every $t \in[0, R]$;

(iv) $\phi_{N}(t) \leq \phi(t)^{N}$ for every $t \in[0, R]$;

(v) $\varphi_{N}$ is a gauge function of order $2 N+1$ on $[0, R]$.

Proof Claim (i) can easily be proved by induction. From (4.2) and (4.3), we get

$$
\phi_{N+1}(t)=\frac{a t^{2} \phi_{N}(t)}{(1-t)(1-b t)-a t^{2} \phi_{N}(t)} \leq \frac{a t^{2} \phi_{N}(t)}{(1-t)(1-b t)-a t^{2}}=\phi(t) \phi_{N}(t),
$$

which proves (ii). Claim (iii) is a trivial consequence from (ii). Claim (iv) follows from (ii) by induction. Claim (v) follows from (i) and the definition of $\varphi_{N}$.

Lemma 4.4 Let $f \in \mathbb{K}[z]$ be a polynomial of degree $n \geq 2, \xi \in \mathbb{K}^{n}$ be a root vector off and $N \geq 0$. Suppose $x \in D_{N}$ is a vector such that $f\left(x_{i}\right) \neq 0$ for some $i \in I_{n}$.

(i) If $x \# T^{(N)}(x)$, then

$$
\frac{f^{\prime}\left(x_{i}\right)}{f\left(x_{i}\right)}-\sum_{j \neq i} \frac{1}{x_{i}-T_{j}^{(N)}(x)}=\frac{1-\sigma_{i}}{x_{i}-\xi_{i}}
$$

where $\sigma_{i} \in \mathbb{K}$ is defined by

$$
\sigma_{i}=\left(x_{i}-\xi_{i}\right) \sum_{j \neq i} \frac{T_{j}^{(N)}(x)-\xi_{j}}{\left(x_{i}-\xi_{j}\right)\left(x_{i}-T_{j}^{(N)}(x)\right)} .
$$

(ii) If $x \in D_{N+1}$, then

$$
T_{i}^{(N+1)}(x)-\xi_{i}=-\frac{\sigma_{i}}{1-\sigma_{i}}\left(x_{i}-\xi_{i}\right) .
$$

Proof (i) Taking into account that $\xi$ is a root vector of $f$, we get

$$
\begin{aligned}
\frac{f^{\prime}\left(x_{i}\right)}{f\left(x_{i}\right)}-\sum_{j \neq i} \frac{1}{x_{i}-T_{j}^{(N)}(x)} & =\sum_{j=1}^{n} \frac{1}{x_{i}-\xi_{j}}-\sum_{j \neq i} \frac{1}{x_{i}-T_{j}^{(N)}(x)} \\
& =\frac{1}{x_{i}-\xi_{i}}+\sum_{j \neq i}\left(\frac{1}{x_{i}-\xi_{j}}-\frac{1}{x_{i}-T_{j}^{(N)}(x)}\right) \\
& =\frac{1}{x_{i}-\xi_{i}}-\sum_{j \neq i} \frac{T_{j}^{(N)}(x)-\xi_{j}}{\left(x_{i}-\xi_{j}\right)\left(x_{i}-T_{j}^{(N)}(x)\right)} \\
& =\frac{1-\sigma_{i}}{x_{i}-\xi_{i}},
\end{aligned}
$$

which proves (4.5).

(ii) It follows from $x \in D_{N+1}$ that

$$
f^{\prime}\left(x_{i}\right)-f\left(x_{i}\right) \sum_{j \neq i} \frac{1}{x_{i}-T_{j}^{(N)}(x)} \neq 0 .
$$


Then from (1.16) and (4.5), we obtain

$$
\begin{aligned}
T_{i}^{(N+1)}(x)-\xi_{i} & =x_{i}-\xi_{i}-\left(\frac{f^{\prime}\left(x_{i}\right)}{f\left(x_{i}\right)}-\sum_{j \neq i} \frac{1}{x_{i}-T_{j}^{(N)}(x)}\right)^{-1} \\
& =x_{i}-\xi_{i}-\frac{x_{i}-\xi_{i}}{1-\sigma_{i}}=-\frac{\sigma_{i}}{1-\sigma_{i}}\left(x_{i}-\xi_{i}\right),
\end{aligned}
$$

which completes the proof.

Lemma 4.5 Let $f \in \mathbb{K}[z]$ be a polynomial of degree $n \geq 2$ which has only simple zeros in $\mathbb{K}, \xi \in \mathbb{K}^{n}$ be a root vector of $f, N \geq 0$, and $1 \leq p \leq \infty$. Suppose $x \in \mathbb{K}^{n}$ is a vector satisfying the following condition:

$$
E(x)=\left\|\frac{x-\xi}{d(\xi)}\right\|_{p}<R=\frac{2}{b+1+\sqrt{(b-1)^{2}+8 a}},
$$

where the function $E: \mathbb{K}^{n} \rightarrow \mathbb{R}_{+}$is defined by (4.1), $a=(n-1)^{1 / q}$, and $b=2^{1 / q}$. Then

$$
x \in D_{N}, \quad\left\|T^{(N)}(x)-\xi\right\| \preceq \phi_{N}(E(x))\|x-\xi\| \quad \text { and } \quad E\left(T^{(N)}(x)\right) \leq \varphi_{N}(E(x)) .
$$

Proof We shall prove statements by induction on $N$. If $N=0$, then (4.10) holds trivially. Assume that (4.10) holds for some $N \geq 0$.

First, we show that $x \in D_{N+1}$, i.e. $x \# T^{(N)}(x)$ and (4.8) holds for every $i \in I_{n}$. It follows from the first inequality in (4.10) that the inequality (3.3) is satisfied with $u=x$ and $v=T^{(N)}(x)$. Then by Lemma 3.3 and (4.9), we obtain

$$
\left|x_{i}-T_{j}^{(N)}(x)\right| \geq\left(1-b\left\|\frac{x-\xi}{d(\xi)}\right\|_{p}\right)\left|\xi_{i}-\xi_{j}\right| \geq(1-b E(x)) d_{j}(\xi)>0
$$

for every $j \neq i$. Consequently, $x \# T^{(N)}(x)$. It remains to prove (4.8) for every $i \in I_{n}$. Let $i \in I_{n}$ be fixed. We shall consider only the non-trivial case $f\left(x_{i}\right) \neq 0$. In this case (4.8) is equivalent to

$$
\frac{f^{\prime}\left(x_{i}\right)}{f\left(x_{i}\right)}-\sum_{j \neq i} \frac{1}{x_{i}-T_{j}^{(N)}(x)} \neq 0 .
$$

We define $\sigma_{i}$ by (4.6). It follows from Lemma 4.4(i) that (4.12) is equivalent to $\sigma_{i} \neq 1$. By Lemma 3.1 with $u=x$ and $v=\xi$ and (4.9), we get

$$
\left|x_{i}-\xi_{j}\right| \geq\left(1-\left\|\frac{x-\xi}{d(\xi)}\right\|_{p}\right)\left|\xi_{i}-\xi_{j}\right| \geq(1-E(x)) d_{i}(\xi)>0
$$

for every $j \neq i$. From the triangle inequality in $\mathbb{K}$, (4.11), (4.13), the induction hypothesis and Hölder's inequality, we get

$$
\begin{aligned}
\left|\sigma_{i}\right| & \leq\left|x_{i}-\xi_{i}\right| \sum_{j \neq i} \frac{\left|T_{j}^{(N)}(x)-\xi_{j}\right|}{\left|x_{i}-\xi_{j}\right|\left|x_{i}-T_{j}^{(N)}(x)\right|} \\
& \leq \frac{1}{(1-E(x))(1-b E(x))} \frac{\left|x_{i}-\xi_{i}\right|}{d_{i}(\xi)} \sum_{j \neq i} \frac{\left|T_{j}^{(N)}(x)-\xi_{j}\right|}{d_{j}(\xi)}
\end{aligned}
$$




$$
\begin{aligned}
& \leq \frac{a E(x) \varphi_{N}(E(x))}{(1-E(x))(1-b E(x))} \\
& =\frac{a E(x)^{2} \phi_{N}(E(x))}{(1-E(x))(1-b E(x))} .
\end{aligned}
$$

From this, $\phi_{N}(E(x)) \leq 1$ and (4.9), we obtain

$$
\left|\sigma_{i}\right| \leq \frac{a E(x)^{2}}{(1-E(x))(1-b E(x))}<1
$$

which yields $\sigma_{i} \neq 1$, and so (4.8) holds. Hence, $x \in D_{N+1}$.

Second, we show that the inequalities in (4.10) hold for $N+1$. The first inequality for $N+1$ is equivalent to

$$
\left|T_{i}^{(N+1)}(x)-\xi_{i}\right| \leq \phi_{N+1}(E(x))\left|x_{i}-\xi_{i}\right| \quad \text { for all } i \in I_{n}
$$

Let $i \in I_{n}$ be fixed. If $x_{i}=\xi_{i}$, then $T_{i}^{(N+1)}(x)=\xi_{i}$ and so (4.15) becomes an equality. Suppose $x_{i} \neq \xi_{i}$. By Lemma 4.4(ii), the triangle inequality in $\mathbb{K}$, and the estimate (4.14), we get

$$
\begin{aligned}
\left|T_{i}^{(N+1)}(x)-\xi_{i}\right| & =\frac{\left|\sigma_{i}\right|}{\left|1-\sigma_{i}\right|}\left|x_{i}-\xi_{i}\right| \leq \frac{\left|\sigma_{i}\right|}{1-\left|\sigma_{i}\right|}\left|x_{i}-\xi_{i}\right| \\
& \leq \frac{a E(x)^{2} \phi_{N}(E(x))}{(1-E(x))(1-b E(x))-a E(x)^{2} \phi_{N}(E(x))}\left|x_{i}-\xi_{i}\right| \\
& =\phi_{N+1}(E(x))\left|x_{i}-\xi_{i}\right|,
\end{aligned}
$$

which proves (4.15). Dividing both sides of the inequality (4.15) by $d_{i}(\xi)$ and taking the p-norm, we obtain

$$
E\left(T^{(N+1)}(x)\right) \leq \varphi_{N+1}(E(x))
$$

which proves that the second inequality in (4.10) holds for $N+1$. This completes the induction and the proof of the lemma.

Now we are ready to state the main result of this section. In the case $N=1$ this result coincides with Theorem 1.1.

Theorem 4.6 Let $f \in \mathbb{K}[z]$ be a polynomial of degree $n \geq 2$ which has only simple zeros in $\mathbb{K}, \xi \in \mathbb{K}^{n}$ be a root vector of $f, N \geq 1$, and $1 \leq p \leq \infty$. Suppose $x^{(0)} \in \mathbb{K}^{n}$ is an initial guess satisfying

$$
E\left(x^{(0)}\right)=\left\|\frac{x^{(0)}-\xi}{d(\xi)}\right\|_{p}<R=\frac{2}{b+1+\sqrt{(b-1)^{2}+8 a}},
$$

where the function $E: \mathbb{K}^{n} \rightarrow \mathbb{R}_{+}$is defined by (4.1), $a=(n-1)^{1 / q}$, and $b=2^{1 / q}$. Then the Ehrlich-type iteration (1.18) is well defined and converges to $\xi$ with error estimates

$$
\left\|x^{(k+1)}-\xi\right\| \preceq \lambda^{(2 N+1)^{k}}\left\|x^{(k)}-\xi\right\| \quad \text { and } \quad\left\|x^{(k)}-\xi\right\| \preceq \lambda^{\left((2 N+1)^{k}-1\right) /(2 N)}\left\|x^{(0)}-\xi\right\|
$$

for all $k \geq 0$, where $\lambda=\phi_{N}\left(E\left(x^{(0)}\right)\right)$ and the function $\phi_{N}$ is defined by Definition 4.1. 
Proof We apply Corollary 2.9 to the iteration function $T^{(N)}: D_{N} \subset \mathbb{K}^{n} \rightarrow \mathbb{K}^{n}$ defined by Definition 1.3 and to the function $E: \mathbb{K}^{n} \rightarrow \mathbb{R}_{+}$defined by (4.1). Let $J=[0, R)$. It follows from Lemma 4.5, Lemma 4.3(v), and Lemma 2.3 that $E$ is a function of the initial conditions of $T^{(N)}$ with a strict gauge function $\varphi_{N}$ of order $r=2 N+1$ on $J$. Since $\xi$ is a root vector of $f$, then $E(\xi)=0 \in J$. It follows from Lemma 4.5 that $T^{(N)}$ is an iterated contraction at a point $\xi$ with respect to $E$ and with control function $\phi_{N}$. The fact that $x^{(0)}$ is an initial point of $T^{(N)}$ follows from Lemma 4.5 and Theorem 2.6. Hence, all the assumptions of Corollary 2.9 are satisfied, and the statement of Theorem 4.6 follows from it.

Corollary 4.7 Let $f \in \mathbb{K}[z]$ be a polynomial of degree $n \geq 2$ which has only simple zeros in $\mathbb{K}, \xi \in \mathbb{K}^{n}$ be a root vector of $f, N \geq 1$, and $1 \leq p \leq \infty$. Suppose $x^{(0)} \in \mathbb{K}^{n}$ is an initial guess satisfying (4.16). Then the Ehrlich-type iteration (1.18) is well defined and converges to $\xi$ with error estimates

$$
\begin{aligned}
& \left\|x^{(k+1)}-\xi\right\| \preceq \lambda^{N(2 N+1)^{k}}\left\|x^{(k)}-\xi\right\| \text { and } \\
& \left\|x^{(k)}-\xi\right\| \preceq \lambda^{\left((2 N+1)^{k}-1\right) / 2}\left\|x^{(0)}-\xi\right\|
\end{aligned}
$$

for all $k \geq 0$, where $\lambda=\phi\left(E\left(x^{(0)}\right)\right)$ and $\phi$ is a real function defined by (1.10).

Proof It follows from Theorem 4.6 and Lemma 4.3(iv).

Let $0<h<1$ be a given number. Solving the equation $\phi(t)=h^{2}$ in the interval $(0, R)$, we can reformulate Corollary 4.7 in the following equivalent form.

Corollary 4.8 Let $f \in \mathbb{K}[z]$ be a polynomial of degree $n \geq 2$ which has $n$ simple zeros in $\mathbb{K}$, $\xi \in \mathbb{K}^{n}$ be a root vector off $, N \geq 1,1 \leq p \leq \infty$, and $0<h<1$. Suppose $x^{(0)} \in \mathbb{K}^{n}$ is an initial guess which satisfies

$$
E\left(x^{(0)}\right)=\left\|\frac{x^{(0)}-\xi}{d(\xi)}\right\|_{p}<R_{h}=\frac{2}{b+1+\sqrt{(b-1)^{2}+4 a\left(1+1 / h^{2}\right)}}
$$

where $a=(n-1)^{1 / q}$ and $b=2^{1 / q}$. Then the Ehrlich-type method (1.18) is well defined and converges to $\xi$ with error estimates

$$
\begin{aligned}
& \left\|x^{(k+1)}-\xi\right\| \preceq h^{2 N(2 N+1)^{k}}\left\|x^{(k)}-\xi\right\| \text { and } \\
& \left\|x^{(k)}-\xi\right\| \preceq h^{(2 N+1)^{k}-1}\left\|x^{(0)}-\xi\right\|
\end{aligned}
$$

for all $k \geq 0$.

Remark 4.9 Corollary 4.8 is an improvement of the result of Kjurkchiev and Andreev [16] (see Theorem 1.4 above). Suppose that a vector $x^{(0)} \in \mathbb{K}^{n}$ satisfies (1.20). It is easy to show that condition (1.19) is equivalent to the following one:

$$
0<c<\min \left\{\frac{\delta}{2(1+(2 n-1) h)}, \frac{2 \delta}{3+2 h+\sqrt{4(3 n-2) h^{2}+4 h+4 n+1}}\right\} .
$$


From this, the initial condition (1.20) and $0<h<1$, we obtain

$$
\begin{aligned}
\left\|\frac{x^{(0)}-\xi}{d(\xi)}\right\|_{\infty} & \leq \frac{\left\|x^{(0)}-\xi\right\|_{\infty}}{\delta} \leq \frac{c h}{\delta} \\
& \leq \frac{2 h}{3+2 h+\sqrt{4(3 n-2) h^{2}+4 h+4 n+1}} \\
& \leq \frac{2}{3+\sqrt{4(3 n-2)+(4 n+1) / h^{2}}} \\
& \leq \frac{2}{3+\sqrt{4 n-3+4(n-1) / h^{2}}} .
\end{aligned}
$$

Therefore, $x^{(0)}$ satisfies (4.19) with $p=\infty$. Then it follows from Corollary 4.8 that the Ehrlich-type method (1.18) is well defined and converges to $\xi$ with error estimates (4.20). From the second estimate in (4.20) and (1.20), we get the estimate (1.21), which completes the proof.

\section{Local convergence theorem of the second type}

Let $f \in \mathbb{K}[z]$ be a polynomial of degree $n \geq 2$. We study the convergence of the Ehrlich-type method (1.18) with respect to the function of the initial conditions $E: \mathcal{D} \rightarrow \mathbb{R}_{+}$defined by

$$
E(x)=\left\|\frac{x-\xi}{d(x)}\right\|_{p} \quad(1 \leq p \leq \infty)
$$

In the previous section, we introduce the functions $\phi_{N}, \varphi_{N}$, and the real number $R$ with two parameters $a>0$ and $b \geq 1$. In this section, we consider a special case of $\phi_{N}, \varphi_{N}$, and $R$ when $b=2$. In other words, now we define $R$ by

$$
R=\frac{2}{3+\sqrt{1+8 a}}
$$

Furthermore, we define the functions $\phi_{N}$ and $\varphi_{N}$ by Definitions 4.1 and 4.2, respectively, but with

$$
\phi_{N+1}(t)=\frac{a t^{2} \phi_{N}(t)}{(1-t)(1-2 t)-a t^{2} \phi_{N}(t)}
$$

instead of (4.2), where $a>0$ is a constant.

Definition 5.1 For a given integer $N \geq 1$, we define the increasing function $\beta_{N}:[0, R] \rightarrow[0,1)$ by

$$
\beta_{N}(t)=\frac{a t^{2} \phi_{N-1}(t)}{1-t-a t^{2} \phi_{N-1}(t)}
$$

and we define the decreasing function $\psi_{N}:[0, R] \rightarrow(0,1]$ as follows:

$$
\psi_{N}(t)=1-2 t\left(1+\beta_{N}(t)\right)=\frac{(1-t)(1-2 t)-a t^{2} \phi_{N-1}(t)}{1-t-a t^{2} \phi_{N-1}(t)} .
$$


Proof of the correctness of Definition 5.1 The functions $\beta_{N}$ and $\psi_{N}$ are well defined on $[0, R]$ since

$$
1-t-a t^{2} \phi_{N-1}(t) \geq 1-t-a t^{2}>0 \quad \text { for all } t \in[0, R]
$$

The monotonicity of $\beta_{N}$ and $\psi_{N}$ is obvious. It remains to prove that $\beta_{N}(R)<1$ and $\psi_{N}(R)>0$. Since $\phi_{N}(R)=1$, we obtain

$$
\beta_{N}(R)=\frac{a R^{2}}{1-R-a R^{2}}<1 \quad \text { and } \quad \psi_{N}(R)=\frac{(1-R)(1-2 R)-a R^{2}}{1-R-a R^{2}}>0,
$$

which completes the proof of the correctness of Definition 5.1.

Lemma 5.2 Let $N \geq 1$. Then:

(i) $\beta_{N}$ is a quasi-homogeneous of degree $2 N$ on $[0, R]$;

(ii) $\beta_{N}(t)=\phi_{N}(t) \psi_{N}(t)$ for every $t \in[0, R]$;

(iii) $\beta_{N+1}(t) \leq \beta_{N}(t)$ for every $t \in[0, R]$;

(iv) $\psi_{N+1}(t) \geq \psi_{N}(t)$ for every $t \in[0, R]$.

Proof The function $\beta_{N}$ can be presented in the form $\beta_{N}(t)=t^{2} \phi_{N-1}(t) \Phi(t)$, where $\Phi(t)=$ $a /\left(1-t-a t^{2} \phi_{N-1}(t)\right)$. Therefore, $\beta_{N}$ is quasi-homogeneous of degree $2 N$ on $[0, R]$ since it is a product of three quasi-homogeneous functions on $[0, R]$ of degree $2,2 N-2$, and 0 . From the definitions of the functions $\phi_{N}, \psi_{N}$, and $\beta_{N}$, we get

$$
\phi_{N}(t) \psi_{N}(t)=\frac{a t^{2} \phi_{N-1}(t)}{(1-t)(1-2 t)-a t^{2} \phi_{N-1}(t)} \frac{(1-t)(1-2 t)-a t^{2} \phi_{N-1}(t)}{1-t-a t^{2} \phi_{N-1}(t)}=\beta_{N}(t) .
$$

Claim (iii) follows from Lemma 4.3(iii) and (5.4). Claim (iv) follows from (iii) and (5.5).

Lemma 5.3 Let $f \in \mathbb{K}[z]$ be a polynomial of degree $n \geq 2$ which splits over $\mathbb{K}, \xi \in \mathbb{K}^{n}$ a root vector off, $N \geq 1$, and $1 \leq p \leq \infty$. Suppose $x \in \mathbb{K}^{n}$ is a vector with distinct components such that

$$
E(x)=\left\|\frac{x-\xi}{d(x)}\right\|_{p} \leq R=\frac{2}{3+\sqrt{1+8 a}}
$$

where the function $E: \mathcal{D} \rightarrow \mathbb{R}_{+}$is defined by (5.1) and $a=(n-1)^{1 / q}$. Then $f$ has only simple zeros in $\mathbb{K}$,

$$
x \in D_{N}, \quad\left\|T^{(N)}(x)-\xi\right\| \preceq \beta_{N}(E(x))\|x-\xi\| \quad \text { and } \quad E\left(T^{(N)}(x)\right) \leq \varphi_{N}(E(x)) .
$$

Besides, the vector $T^{(N)}(x)$ has pairwise distinct components.

Proof It follows from (5.7) and $R<1 / 2$ that $E(x)<1 / 2$. Then it follows from Lemma 3.2 that the vector $\xi$ has distinct components, which means that $f$ has only simple zeros in $\mathbb{K}$. We divide the proof into two steps.

Step 1. In this step, we prove $x \in D_{N}$ and the first inequality in (5.8) by induction on $N$. If $N=1$, the proof of the claims can be found in [11]. Assume that $x \in D_{N}$ and the first inequality in (5.8) hold for some $N \geq 1$. 
First we show that $x \in D_{N+1}$, i.e. $x \# T^{(N)}(x)$ and (4.8) holds for every $i \in I_{n}$. It follows from the first inequality in (5.8) that (3.5) holds with $v=x, u=T^{(N)}(x)$, and $\alpha=1$. Therefore by Lemma 3.4, (5.7) and $R<1 / 2$, we obtain

$$
\left|x_{i}-T_{j}^{(N)}(x)\right|>\left(1-2\left\|\frac{x-\xi}{d(x)}\right\|_{p}\right)\left|x_{i}-x_{j}\right| \geq(1-2 E(x)) d_{j}(x)>0
$$

for every $j \neq i$. Consequently, $x \# T^{(N)}(x)$. It remains to prove (4.8) for every $i \in I_{n}$. Let $i \in I_{n}$ be fixed. We shall consider only the non-trivial case $f\left(x_{i}\right) \neq 0$. In this case (4.8) is equivalent to (4.12). On the other hand, it follows from Lemma 4.4(i) that (4.12) is equivalent to $\sigma_{i} \neq 1$, where $\sigma_{i}$ is defined by (4.6). By Lemma 3.1 with $u=\xi$ and $v=x$ and (5.7), we get

$$
\left|x_{i}-\xi_{j}\right| \geq\left(1-\left\|\frac{x-\xi}{d(x)}\right\|_{p}\right)\left|x_{i}-x_{j}\right|=(1-E(x))\left|x_{i}-x_{j}\right| \geq(1-E(x)) d_{i}(x)>0
$$

for every $j \neq i$. Hence, we obtain $x \# \xi$. From the induction hypothesis, we get

$$
\left|T_{i}^{(N)}(x)-\xi_{i}\right| \leq \beta_{N}(E(x))\left|x_{i}-\xi_{i}\right|
$$

Combining the triangle inequality in $\mathbb{K},(5.10)$, (5.9) and (5.11), we obtain

$$
\begin{aligned}
\left|\sigma_{i}\right| & \leq\left|x_{i}-\xi_{i}\right| \sum_{j \neq i} \frac{\left|T_{j}^{(N)}(x)-\xi_{j}\right|}{\left|x_{i}-\xi_{j}\right|\left|x_{i}-T_{j}^{(N)}(x)\right|} \\
& \leq \frac{1}{(1-E(x))(1-2 E(x))} \frac{\left|x_{i}-\xi_{i}\right|}{d_{i}(x)} \sum_{j \neq i} \frac{\left|T_{j}^{(N)}(x)-\xi_{j}\right|}{d_{j}(x)} \\
& \leq \frac{\beta_{N}(E(x))}{(1-E(x))(1-2 E(x))} \frac{\left|x_{i}-\xi_{i}\right|}{d_{i}(x)} \sum_{j \neq i} \frac{\left|x_{j}-\xi_{j}\right|}{d_{j}(x)}
\end{aligned}
$$

which, using Hölder's inequality, yields

$$
\left|\sigma_{i}\right| \leq \frac{a E(x)^{2} \beta_{N}(E(x))}{(1-E(x))(1-2 E(x))}
$$

From this and (5.7), we deduce

$$
\left|\sigma_{i}\right| \leq \frac{a E(x)^{2}}{(1-E(x))(1-2 E(x))}<1,
$$

which yields $\sigma_{i} \neq 1$, and so (4.12) holds. Thus we prove that $x \in D_{N+1}$.

Now we have to prove that the first inequality in (5.8) is satisfied for $N+1$, which is equivalent to

$$
\left|T_{i}^{(N+1)}(x)-\xi_{i}\right| \leq \beta_{N+1}(E(x))\left|x_{i}-\xi_{i}\right| \quad \text { for all } i \in I_{n}
$$

Let $i \in I_{n}$ be fixed. If $x_{i}=\xi_{i}$, then $T_{i}^{(N+1)}(x)=\xi_{i}$ and the inequality (5.13) becomes an equality. Suppose $x_{i} \neq \xi_{i}$. It follows from Lemma 4.4(ii), the triangle inequality in $\mathbb{K}$, and the 
estimate (5.12) that

$$
\begin{aligned}
\left|T_{i}^{(N+1)}(x)-\xi_{i}\right| & =\frac{\left|\sigma_{i}\right|}{\left|1-\sigma_{i}\right|}\left|x_{i}-\xi_{i}\right| \leq \frac{\left|\sigma_{i}\right|}{1-\left|\sigma_{i}\right|}\left|x_{i}-\xi_{i}\right| \\
& \leq \frac{a E(x)^{2} \beta_{N}(E(x))}{(1-E(x))(1-2 E(x))-a E(x)^{2} \beta_{N}(E(x))}\left|x_{i}-\xi_{i}\right| .
\end{aligned}
$$

From this inequality, Lemma 5.2(ii), $\psi_{N}(t) \leq 1$, (5.3) and Lemma 5.2(iv), we obtain

$$
\begin{aligned}
\left|T_{i}^{(N+1)}(x)-\xi_{i}\right| & \leq \frac{a E(x)^{2} \phi_{N}(E(x)) \psi_{N}(E(x))}{(1-E(x))(1-2 E(x))-a E(x)^{2} \phi_{N}(E(x))}\left|x_{i}-\xi_{i}\right| \\
& \leq \phi_{N+1}(E(x)) \psi_{N}(E(x))\left|x_{i}-\xi_{i}\right| \\
& \leq \phi_{N+1}(E(x)) \psi_{N+1}(E(x))\left|x_{i}-\xi_{i}\right|=\beta_{N+1}(E(x))\left|x_{i}-\xi_{i}\right|
\end{aligned}
$$

which proves (5.13). This completes the induction.

Step 2. In this step we prove the second inequality in (5.8) and that $T^{(N)}(x)$ has distinct components. First inequality in (5.8) allow us to apply Lemma 3.5 with $u=T^{(N)}(x), v=x$, and $\alpha=\beta_{N}(E(x))$. By Lemma 3.5 and (5.5), we deduce

$$
\left|T_{i}^{(N)}(x)-T_{j}^{(N)}(x)\right| \geq\left(1-2^{1 / q} E(x)\left(1+\beta_{N}(E(x))\right)\right)\left|x_{i}-x_{j}\right| \geq \psi_{N}(E(x))\left|x_{i}-x_{j}\right| .
$$

By taking the minimum over all $j \in I_{n}$ such that $j \neq i$, we obtain

$$
d_{i}\left(T^{(N)}(x)\right) \geq \psi_{N}(E(x)) d_{i}(x)>0
$$

which implies that $T^{(N)}(x)$ has distinct components. It follows from (5.11), (5.14), and Lemma 5.2(ii) that

$$
\frac{\left|T_{i}^{(N)}(x)-\xi_{i}\right|}{d_{i}\left(T^{(N)}(x)\right)} \leq \frac{\beta_{N}(E(x))}{\psi_{N}(E(x))} \frac{\left|x_{i}-\xi_{i}\right|}{d_{i}(x)}=\phi_{N}(E(x)) \frac{\left|x_{i}-\xi_{i}\right|}{d_{i}(x)} .
$$

By taking the $p$-norm, we obtain

$$
E\left(T^{(N)}(x)\right) \leq \phi_{N}(E(x)) E(x)=\varphi_{N}(E(x))
$$

which proves the second inequality in (5.8). This completes the proof.

Now we are able to state the main result of this section. In the case when $N=1$ and $p=\infty$ this result reduces to Theorem 1.2.

Theorem 5.4 Let $f \in \mathbb{K}[z]$ be a polynomial of degree $n \geq 2$ which splits over $\mathbb{K}, \xi \in \mathbb{K}^{n}$ be a root vector off,$N \geq 1$, and $1 \leq p \leq \infty$. Suppose $x^{(0)} \in \mathbb{K}^{n}$ is an initial guess with distinct components such that

$$
E\left(x^{(0)}\right)=\left\|\frac{x^{(0)}-\xi}{d\left(x^{(0)}\right)}\right\|_{p} \leq R=\frac{2}{3+\sqrt{1+8 a}},
$$


where the function $E$ is defined by (5.1) and $a=(n-1)^{1 / q}$. Then $f$ has only simple zeros in $\mathbb{K}$, and the Ehrlich-type iteration (1.18) is well defined and converges to $\xi$ with error estimates

$$
\begin{aligned}
& \left\|x^{(k+1)}-\xi\right\| \preceq \theta \lambda^{(2 N+1)^{k}}\left\|x^{(k)}-\xi\right\| \quad \text { and } \\
& \left\|x^{(k)}-\xi\right\| \preceq \theta^{k} \lambda^{\left((2 N+1)^{k}-1\right) / 2 N}\left\|x^{(0)}-\xi\right\|
\end{aligned}
$$

for all $k \geq 0$, where $\lambda=\phi_{N}\left(E\left(x^{(0)}\right)\right), \theta=\psi_{N}\left(E\left(x^{(0)}\right)\right)$. Moreover, the method is convergent with order $2 N+1$ provided that $E\left(x^{(0)}\right)<R$.

Proof We apply Theorem 2.8 to the iteration function $T^{(N)}: D_{N} \subset \mathbb{K}^{n} \rightarrow \mathbb{K}^{n}$ together with the function $E: D_{N} \rightarrow \mathbb{R}_{+}$defined by (5.1).

It follows from Lemma 5.3 and Lemma 4.3(v) that $E$ is a function of the initial conditions of $T^{(N)}$ with gauge function $\varphi_{N}$ of order $r=2 N+1$ on the interval $J=[0, R]$.

From Lemma 5.3, we see that $T^{(N)}$ is an iterated contraction at $\xi$ with respect to $E$ and with control function $\beta_{N}$. Also, it is easy to see that the functions $\beta_{N}, \phi_{N}, \psi_{N}$, and $\varphi_{N}$ have the properties (2.5), (2.6) and (2.7).

It follows from Lemma 5.3 that $x^{(0)} \in D_{N}$. According to Theorem 2.6 to prove that $x^{(0)}$ is an initial point of $T^{(N)}$ it is sufficient to prove that

$$
x \in D_{N} \quad \text { and } \quad E(x) \in J \quad \Rightarrow \quad T^{(N)}(x) \in D_{N} .
$$

From $x \in D_{N}$, we have $T^{(N)}(x) \in \mathbb{K}^{n}$. By Lemma 5.3, $T^{(N)}(x)$ has distinct components and $E\left(T^{(N)}(x)\right) \leq \varphi_{N}(E(x))$. The last inequality yields $E\left(T^{(N)}(x)\right) \in J$ since $\varphi_{N}: J \rightarrow J$ and $E(x) \in J$. Thus we have both $T^{(N)}(x) \in \mathcal{D}$ and $E\left(T^{(N)}(x)\right) \in J$. Applying Lemma 5.3 to the vector $T^{(N)}(x)$, we get $T^{(N)}(x) \in D_{N}$, which proves (5.17). Therefore, $x^{(0)}$ is an initial point of $T^{(N)}$.

Now the statement of Theorem 5.4 follows from Theorem 2.8.

\section{Semilocal convergence theorem}

In this section we establish semilocal convergence theorems for Ehrlich-type methods (1.18) for finding all zeros of a polynomial simultaneously. We study the convergence of these methods with respect to the function of the initial conditions $E_{f}: \mathcal{D} \rightarrow \mathbb{R}_{+}$defined by

$$
E_{f}(x)=\left\|\frac{W_{f}(x)}{d(x)}\right\|_{p} \quad(1 \leq p \leq \infty) .
$$

Recently Proinov [22] has shown that there is a relationship between local and semilocal theorems for simultaneous root-finding methods. It turns out that from any local convergence theorem for a simultaneous method one can obtain as a consequence a semilocal theorem for the same method. In particular, from Theorem 5.4 we can obtain a semilocal convergence theorem for Ehrlich-type methods (1.18) under computationally verifiable initial conditions. For this purpose we need the following result.

Theorem 6.1 (Proinov [22]) Let $f \in \mathbb{K}[z]$ be a polynomial of degree $n \geq 2$. Suppose $x \in \mathbb{K}^{n}$ is an initial guess with distinct components such that

$$
\left\|\frac{W_{f}(x)}{d(x)}\right\|_{p} \leq \frac{R(1-R)}{1+(a-1) R}
$$


for some $1 \leq p \leq \infty$ and $0<R \leq 1 /(1+\sqrt{a})$, where $a=(n-1)^{1 / q}$. In the case $n=2$ and $p=\infty$ we assume that inequality in (6.2) is strict. Then $f$ has only simple zeros in $\mathbb{K}$, and there exists a root vector $\xi \in \mathbb{K}^{n}$ off such that

$$
\|x-\xi\| \preceq \alpha\left(E_{f}(x)\right)\left\|W_{f}(x)\right\| \quad \text { and } \quad\left\|\frac{x-\xi}{d(x)}\right\|_{p} \leq R
$$

where the real function $\alpha$ is defined by

$$
\alpha(t)=\frac{2}{1-(a-1) t+\sqrt{(1-(a-1) t)^{2}-4 t}} .
$$

If the inequality (6.2) is strict, then the second inequality in (6.3) is strict too.

Now, we are ready to state and prove the main result of this paper.

Theorem 6.2 Let $f \in \mathbb{K}[z]$ be a polynomial of degree $n \geq 2, N \geq 1,1 \leq p \leq \infty$. Suppose $x^{(0)} \in \mathbb{K}^{n}$ is an initial guess with distinct components such that

$$
E_{f}\left(x^{(0)}\right)=\left\|\frac{W_{f}\left(x^{(0)}\right)}{d\left(x^{(0)}\right)}\right\|_{p}<\frac{8}{(3+\sqrt{1+8 a})^{2}}
$$

where the function $E_{f}$ is defined by $(6.1)$ and $a=(n-1)^{1 / q}$. Then $f$ has only simple zeros in $\mathbb{K}$, and the Ehrlich-type iteration (1.18) is well defined and converges to a root vector $\xi$ off with order of convergence $2 N+1$ and with an a posteriori error estimate

$$
\left\|x^{(k)}-\xi\right\| \preceq \alpha\left(E_{f}\left(x^{(k)}\right)\right)\left\|W_{f}\left(x^{(k)}\right)\right\|
$$

for all $k \geq 0$ such that $E_{f}\left(x^{(k)}\right)<8 /(3+\sqrt{1+8 a})^{2}$, where the function $\alpha$ is defined by (6.4).

Proof Let us define $R$ by (5.2). It is easy to calculate that $R<1 /(1+\sqrt{a})$ and

$$
\frac{R(1-R)}{1+(a-1) R}=\frac{2(1+\sqrt{1+8 a})}{(3+\sqrt{1+8 a})(1+2 a+\sqrt{1+8 a})}=\frac{8}{(3+\sqrt{1+8 a})^{2}} .
$$

Therefore, (6.5) can be written in the form

$$
\left\|\frac{W_{f}\left(x^{(0)}\right)}{d\left(x^{(0)}\right)}\right\|_{p}<\frac{R(1-R)}{1+(a-1) R}
$$

Then it follows from Theorem 6.1 that $f$ has only simple zeros in $\mathbb{K}$ and there exists a root vector $\xi \in \mathbb{K}^{n}$ of $f$ such that

$$
\left\|\frac{x^{(0)}-\xi}{d\left(x^{(0)}\right)}\right\|_{p}<R
$$

Now Theorem 5.4 implies that the Ehrlich-type iteration (1.18) converges to $\xi$ with order of convergence $2 N+1$. It remains to prove the error estimate (6.6). Suppose that for some 
$k \geq 0$,

$$
\left\|\frac{W_{f}\left(x^{(k)}\right)}{d\left(x^{(k)}\right)}\right\|_{p}<\frac{R(1-R)}{1+(a-1) R} .
$$

Then it follows from Theorem 6.1 that there exists a root vector $\eta \in \mathbb{K}^{n}$ of $f$ such that

$$
\left\|x^{(k)}-\eta\right\| \preceq \alpha\left(E_{f}\left(x^{(k)}\right)\right)\left\|W_{f}\left(x^{(k)}\right)\right\| \quad \text { and } \quad\left\|\frac{x^{(k)}-\eta}{d\left(x^{(k)}\right)}\right\|_{p}<R \text {. }
$$

From the second inequality in (6.8) and Theorem 5.4, we conclude that the Ehrlich-type iteration (1.18) converges to $\eta$. By the uniqueness of the limit, we get $\eta=\xi$. Therefore, the error estimate (6.6) follows from the first inequality in (6.8). This completes the proof.

Setting $p=\infty$ in Theorem 6.2 , we obtain the following result.

Corollary 6.3 Let $f \in \mathbb{K}[z]$ be a polynomial of degree $n \geq 2$ and $N \geq 1$. Suppose $x^{(0)} \in \mathbb{K}^{n}$ is an initial guess with distinct components such that

$$
\left\|\frac{W_{f}\left(x^{(0)}\right)}{d\left(x^{(0)}\right)}\right\|_{\infty}<\frac{8}{(3+\sqrt{8 n-7})^{2}} .
$$

Then $f$ has only simple zeros in $\mathbb{K}$ and the Ehrlich-type iteration (1.18) is well defined and converges to a root vector $\xi$ of $f$ with order of convergence $2 N+1$ and with error estimate (6.6) for $p=\infty$.

Setting $p=1$ in Theorem 6.2 we obtain the following result.

Corollary 6.4 Let $f \in \mathbb{K}[z]$ be a polynomial of degree $n \geq 2$ and $N \geq 1$. Suppose $x^{(0)} \in \mathbb{K}^{n}$ is an initial guess with distinct components such that

$$
\left\|\frac{W_{f}\left(x^{(0)}\right)}{d\left(x^{(0)}\right)}\right\|_{1}<\frac{2}{9} .
$$

Then $f$ has only simple zeros in $\mathbb{K}$ and the Ehrlich-type iteration (1.18) is well defined and converges with order $2 N+1$ to a root vector $\xi$ off with error estimate (6.6) for $p=1$.

\section{Numerical examples}

In this section, we present several numerical examples to show some applications of Theorem 6.2. Let $f \in \mathbb{C}[z]$ be a polynomial of degree $n \geq 2$ and let $x^{(0)} \in \mathbb{C}^{n}$ be an initial guess. We show that Theorem 6.2 can be used:

- to prove numerically that $f$ has only simple zeros;

- to prove numerically that $N$ th Ehrlich-type iteration (1.18) starting from $x^{(0)}$ is well defined and converges with order $2 N+1$ to a root vector of $f$;

- to guarantee the desired accuracy when calculating the roots of $f$ via the $N$ th Ehrlich-type method.

In the examples below, we use the function of the initial conditions $E_{f}: \mathcal{D} \rightarrow \mathbb{R}_{+}$defined by

$$
E_{f}(x)=\left\|\frac{W_{f}(x)}{d(x)}\right\|_{\infty},
$$


where $W_{f}$ is the Weierstrass correction defined by (1.6). We consider only the case $p=\infty$, since the other cases are similar.

Also, we use the real function $\alpha$ defined by

$$
\alpha(t)=\frac{2}{1-(n-2) t+\sqrt{(1-(n-2) t)^{2}-4 t}} .
$$

It follows from Theorem 6.2 that if there exists an integer $m \geq 0$ such that

$$
E_{f}\left(x^{(m)}\right) \leq \mathscr{R}=\frac{8}{(3+\sqrt{8 n-7})^{2}},
$$

then $f$ has only simple zeros and the Ehrlich-type iteration (1.18) is well defined and converges to a root vector $\xi$ of $f$ with order of convergence $2 N+1$. Besides, for all $k \geq m$ such that

$$
E_{f}\left(x^{(k)}\right)<\mathscr{R}=\frac{8}{(3+\sqrt{8 n-7})^{2}}
$$

the following a posteriori error estimate holds:

$$
\left\|x^{(k)}-\xi\right\|_{\infty}<\varepsilon_{k}, \quad \text { where } \varepsilon_{k}=\alpha\left(E_{f}\left(x^{(k)}\right)\right)\left\|W_{f}\left(x^{(k)}\right)\right\|_{\infty} .
$$

In the examples, we apply the Ehrlich-type methods (1.18) for some $N \geq 1$ using the following stopping criterion:

$$
\varepsilon_{k}<10^{-15} \quad \text { and } \quad E_{f}\left(x^{(k)}\right)<\mathscr{R} \quad(k \geq m) .
$$

For given $N$ we calculate the smallest $m \geq 0$ which satisfies the convergence condition (7.3), the smallest $k \geq m$ for which the stopping criterion (7.6) is satisfied, as well as the value of $\varepsilon_{k}$ for the last $k$.

In Table 2 the values of iterations are given to 15 decimal places. The values of other quantities $\left(\mathscr{R}, E_{f}\left(x^{(m)}\right)\right.$, etc.) are given to six decimal places.

Numerical calculations are made using the software package Mathematica [23].

Example 7.1 We consider the polynomial

$$
f(z)=z^{4}-1
$$

and the initial guess

$$
x^{(0)}=(0.5+0.5 i,-1.36+0.42 i,-0.25+1.28 i, 0.46-1.37 i)
$$

which are taken from Zhang et al. [24]. We have $\mathscr{R}=0.125$ and $E\left(x^{(0)}\right)=0.506619$. The results for this example are presented in Table 1 . For example, we can see that for $N=10$ at the first iteration we have proved that the Ehrlich-type method converges with order of convergence 21 and that at the second iteration we have calculated the zeros $f$ with accuracy less than $10^{-127}$. Moreover, at the next iteration we obtain the zeros of $f$ with accuracy less than $10^{-2,682}$. Also, we can see that for $N=100$ at the second iteration we have obtained the zeros of $f$ with accuracy less than $10^{-11,450}$. 
Table 1 Values of $m, k$, and $\varepsilon_{k}$ for Example $7.1(\mathscr{R}=0.125)$

\begin{tabular}{rllllll}
\hline $\boldsymbol{N}$ & $\boldsymbol{m}$ & $\boldsymbol{E}_{\boldsymbol{f}}\left(\boldsymbol{x}^{(\boldsymbol{m})}\right)$ & $\boldsymbol{\varepsilon}_{\boldsymbol{m}}$ & $\boldsymbol{k}$ & $\boldsymbol{\varepsilon}_{\boldsymbol{k}}$ & $\boldsymbol{\varepsilon}_{\boldsymbol{k}+\mathbf{1}}$ \\
\hline 1 & 2 & 0.010032 & $1.457548 \times 10^{-2}$ & 4 & $4.385760 \times 10^{-21}$ & $8.919073 \times 10^{-63}$ \\
2 & 1 & 0.067725 & $1.242914 \times 10^{-1}$ & 3 & $1.347060 \times 10^{-38}$ & $7.284576 \times 10^{-193}$ \\
3 & 1 & 0.015716 & $2.300541 \times 10^{-2}$ & 3 & $1.825502 \times 10^{-106}$ & $5.054741 \times 10^{-744}$ \\
4 & 1 & 0.002730 & $3.887455 \times 10^{-3}$ & 2 & $1.330837 \times 10^{-25}$ & $3.543773 \times 10^{-230}$ \\
5 & 1 & 0.001215 & $1.722883 \times 10^{-3}$ & 2 & $4.720064 \times 10^{-37}$ & $2.999643 \times 10^{-407}$ \\
6 & 1 & 0.000206 & $2.927439 \times 10^{-4}$ & 2 & $1.060096 \times 10^{-50}$ & $5.523501 \times 10^{-657}$ \\
7 & 1 & 0.000081 & $1.155284 \times 10^{-4}$ & 2 & $6.261239 \times 10^{-67}$ & $3.252761 \times 10^{-1,002}$ \\
8 & 1 & 0.000014 & $1.986052 \times 10^{-5}$ & 2 & $6.080606 \times 10^{-85}$ & $3.570038 \times 10^{-1,439}$ \\
9 & 1 & 0.000005 & $7.910775 \times 10^{-6}$ & 2 & $1.309022 \times 10^{-105}$ & $1.170454 \times 10^{-2,002}$ \\
10 & 1 & 0.000000 & $1.366899 \times 10^{-6}$ & 2 & $4.301615 \times 10^{-128}$ & $8.477451 \times 10^{-2,683}$ \\
100 & 1 & 0.000000 & $1.820743 \times 10^{-57}$ & 1 & $1.820743 \times 10^{-57}$ & $3.460397 \times 10^{-11,451}$ \\
\hline
\end{tabular}

Table 2 Numerical results for Example 7.1 in the case $N=10$

\begin{tabular}{lll}
\hline $\boldsymbol{k}$ & $\boldsymbol{x}_{\mathbf{1}}^{(\boldsymbol{k})}$ & $\boldsymbol{x}_{\mathbf{2}}^{(\boldsymbol{k})}$ \\
\hline 0 & $0.5+0.5 i$ & $-1.36+0.42 i$ \\
1 & $1.000000380419496+0.000000816235730 i$ & $-1.000000220051461-0.000000495915480 i$ \\
2 & $1.000000000000000+0.000000000000000 i$ & $-1.000000000000000+0.000000000000000 i$ \\
\hline $\boldsymbol{k}$ & $\boldsymbol{x}_{\mathbf{1}}^{(\boldsymbol{k})}$ & $\boldsymbol{x}_{\mathbf{2}}^{(\boldsymbol{k})}$ \\
\hline 0 & $-0.25+1.28 i$ & $0.46-1.37 i$ \\
1 & $0.000000277962637+0.999999578393062 i$ & $-0.000000314533436-0.999998669784542 i$ \\
2 & $0.000000000000000+1.000000000000000 i$ & $0.000000000000000-1.000000000000000 i$ \\
\hline
\end{tabular}

Table 3 Values of $m, k$, and $\varepsilon_{k}$ for Example $7.2(\mathscr{R}=0.043061)$

\begin{tabular}{rllllll}
\hline $\boldsymbol{N}$ & $\boldsymbol{m}$ & $\boldsymbol{E}_{\boldsymbol{f}}\left(\boldsymbol{x}^{(\boldsymbol{m})}\right)$ & $\boldsymbol{\varepsilon}_{\boldsymbol{m}}$ & $\boldsymbol{k}$ & $\boldsymbol{\varepsilon}_{\boldsymbol{k}}$ & $\boldsymbol{\varepsilon}_{\boldsymbol{k}+\mathbf{1}}$ \\
\hline 1 & 6 & 0.036897 & $3.187918 \times 10^{-2}$ & 9 & $3.967908 \times 10^{-36}$ & $5.304009 \times 10^{-106}$ \\
2 & 5 & 0.000003 & $1.182714 \times 10^{-6}$ & 6 & $6.112531 \times 10^{-28}$ & $2.230412 \times 10^{-134}$ \\
3 & 4 & 0.000064 & $2.475020 \times 10^{-5}$ & 5 & $2.446120 \times 10^{-29}$ & $2.722168 \times 10^{-197}$ \\
4 & 4 & 0.000000 & $1.550670 \times 10^{-11}$ & 5 & $3.838741 \times 10^{-93}$ & $1.589981 \times 10^{-827}$ \\
5 & 3 & 0.005793 & $2.415745 \times 10^{-3}$ & 4 & $9.532339 \times 10^{-24}$ & $8.487351 \times 10^{-248}$ \\
6 & 3 & 0.000293 & $1.127450 \times 10^{-4}$ & 4 & $9.565008 \times 10^{-45}$ & $1.725858 \times 10^{-565}$ \\
7 & 3 & 0.000005 & $2.173198 \times 10^{-6}$ & 4 & $4.018844 \times 10^{-77}$ & $6.737932 \times 10^{-1,138}$ \\
8 & 3 & 0.000000 & $1.562375 \times 10^{-8}$ & 4 & $1.162424 \times 10^{-123}$ & $1.291370 \times 10^{-2,080}$ \\
9 & 3 & 0.000000 & $4.092421 \times 10^{-11}$ & 4 & $4.245137 \times 10^{-187}$ & $1.373908 \times 10^{-3,530}$ \\
10 & 3 & 0.000000 & $3.904607 \times 10^{-14}$ & 4 & $4.643262 \times 10^{-270}$ & $2.543247 \times 10^{-5,644}$ \\
30 & 2 & 0.000055 & $2.129417 \times 10^{-5}$ & 3 & $5.721566 \times 10^{-249}$ & $2.377023 \times 10^{-15,106}$ \\
\hline
\end{tabular}

In Table 2, we present numerical results for Example 7.1 in the case $N=10$.

Example 7.2 We consider the polynomial

$$
f(z)=z^{15}+z^{14}+1
$$

and Aberth's initial approximation $x^{(0)} \in \mathbb{C}^{n}$ given by (see Aberth [8] and Petković et al. $[25])$ :

$$
x_{v}^{(0)}=-\frac{a_{1}}{n}+r_{0} \exp \left(i \theta_{v}\right), \quad \theta_{v}=\frac{\pi}{n}\left(2 v-\frac{3}{2}\right), v=1, \ldots, n,
$$

where $a_{1}=1, r_{0}=2$, and $n=15$. We have $\mathscr{R}=0.043061$ and $E\left(x^{(0)}\right)=0.179999$. The results for this example are presented in Table 3. For example, we can see that for $N=30$ at the 
Table 4 Values of $m, k$, and $\varepsilon_{k}$ for Example $7.3(\mathscr{R}=0.033867)$

\begin{tabular}{rllllll}
\hline $\boldsymbol{N}$ & $\boldsymbol{m}$ & $\boldsymbol{E}_{\boldsymbol{f}}\left(\boldsymbol{x}^{(\boldsymbol{m})}\right)$ & $\boldsymbol{\varepsilon}_{\boldsymbol{m}}$ & $\boldsymbol{k}$ & $\boldsymbol{\varepsilon}_{\boldsymbol{k}}$ & $\boldsymbol{\varepsilon}_{\boldsymbol{k}+\mathbf{1}}$ \\
\hline 1 & 18 & 0.000060 & $6.095859 \times 10^{-5}$ & 20 & $1.620028 \times 10^{-38}$ & $4.276235 \times 10^{-114}$ \\
2 & 12 & 0.015335 & $2.153155 \times 10^{-2}$ & 14 & $1.095084 \times 10^{-46}$ & $1.779476 \times 10^{-230}$ \\
3 & 10 & 0.018005 & $2.769333 \times 10^{-2}$ & 12 & $8.917532 \times 10^{-86}$ & $4.482714 \times 10^{-596}$ \\
4 & 9 & 0.005514 & $6.130790 \times 10^{-3}$ & 10 & $4.221856 \times 10^{-21}$ & $7.250879 \times 10^{-184}$ \\
5 & 9 & 0.000000 & $1.159694 \times 10^{-15}$ & 10 & $5.021359 \times 10^{-165}$ & $5.118016 \times 10^{-1,808}$ \\
6 & 8 & 0.000237 & $2.386016 \times 10^{-4}$ & 9 & $8.455240 \times 10^{-48}$ & $1.280870 \times 10^{-612}$ \\
7 & 8 & 0.000000 & $2.723047 \times 10^{-17}$ & 8 & $2.723047 \times 10^{-17}$ & $8.926059 \times 10^{-249}$ \\
8 & 7 & 0.018995 & $2.934241 \times 10^{-2}$ & 8 & $2.885374 \times 10^{-30}$ & $4.152134 \times 10^{-503}$ \\
9 & 7 & 0.002180 & $2.274734 \times 10^{-3}$ & 8 & $3.792876 \times 10^{-51}$ & $1.140751 \times 10^{-958}$ \\
10 & 7 & 0.000000 & $5.185525 \times 10^{-7}$ & 8 & $1.620086 \times 10^{-132}$ & $2.936276 \times 10^{-2,768}$ \\
30 & 5 & 0.000181 & $1.821419 \times 10^{-4}$ & 6 & $1.395923 \times 10^{-226}$ & $1.902920 \times 10^{-13,777}$ \\
\hline & & & & & &
\end{tabular}

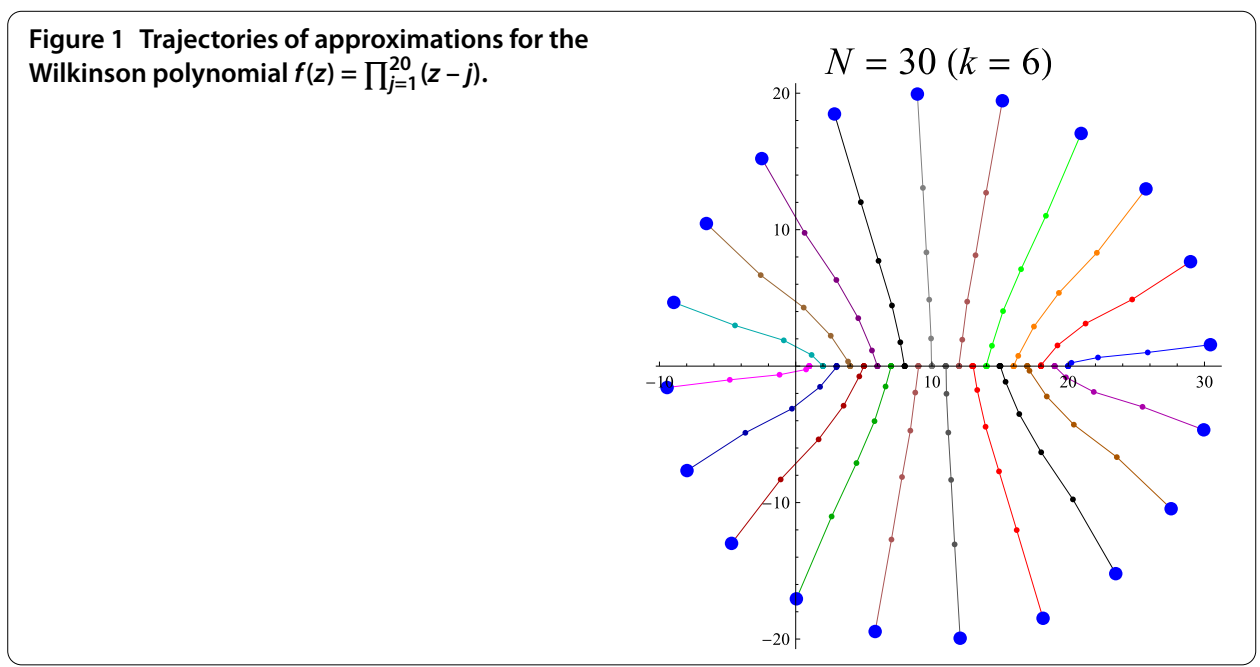

third iteration we have obtained the zeros of $f$ with accuracy less than $10^{-248}$. Moreover, at the next iteration we get the zeros of $f$ with accuracy less than $10^{-15,105}$.

Example 7.3 We consider the Wilkinson polynomial [26]

$$
f(z)=\prod_{j=1}^{20}(z-j)=z^{20}-120 z^{14}+\cdots+2,432,902,008,176,640,000
$$

and Aberth's initial approximation (7.7) with $a_{1}=-120, r_{0}=20$, and $n=20$. We have $\mathscr{R}=0.033867$ and $E\left(x^{(0)}\right)=0.344409$. The results for Example 7.3 are shown in Table 4 . For example, for $N=30$ at the seventh iteration, we get the zeros of $f$ with accuracy less than $10^{-13,776}$.

In Figure 1, we present the trajectories of approximations generated by the method (1.18) for $N=30$ after 6 iterations.

Example 7.4 We consider the polynomial

$$
f(z)=z^{40}-1
$$


Table 5 Values of $m, k$, and $\varepsilon_{k}$ for Example $7.4(\mathscr{R}=\mathbf{0 . 0 1 8 6 8 5 )}$

\begin{tabular}{rrlllll}
\hline $\boldsymbol{N}$ & $\boldsymbol{m}$ & $\boldsymbol{E}_{\boldsymbol{f}}\left(\boldsymbol{x}^{(\boldsymbol{m})}\right)$ & $\boldsymbol{\varepsilon}_{\boldsymbol{m}}$ & $\boldsymbol{k}$ & $\boldsymbol{\varepsilon}_{\boldsymbol{k}}$ & $\boldsymbol{\varepsilon}_{\boldsymbol{k}+\mathbf{1}}$ \\
\hline 1 & 15 & 0.007235 & $1.588799 \times 10^{-3}$ & 17 & $1.057241 \times 10^{-18}$ & $1.574672 \times 10^{-52}$ \\
2 & 11 & 0.000001 & $1.731641 \times 10^{-7}$ & 12 & $2.763909 \times 10^{-30}$ & $2.863869 \times 10^{-144}$ \\
3 & 9 & 0.000026 & $4.171842 \times 10^{-6}$ & 10 & $5.167701 \times 10^{-32}$ & $2.328540 \times 10^{-213}$ \\
4 & 8 & 0.000032 & $5.141616 \times 10^{-6}$ & 9 & $7.830010 \times 10^{-40}$ & $3.487627 \times 10^{-344}$ \\
5 & 7 & 0.010766 & $2.954474 \times 10^{-3}$ & 8 & $1.468181 \times 10^{-20}$ & $2.870206 \times 10^{-208}$ \\
6 & 7 & 0.000002 & $4.201055 \times 10^{-7}$ & 8 & $7.096655 \times 10^{-71}$ & $6.481892 \times 10^{-900}$ \\
7 & 7 & 0.000000 & $9.445503 \times 10^{-15}$ & 8 & $3.169914 \times 10^{-196}$ & $2.445585 \times 10^{-2,918}$ \\
8 & 6 & 0.010675 & $2.911647 \times 10^{-3}$ & 7 & $8.218559 \times 10^{-31}$ & $3.538870 \times 10^{-495}$ \\
9 & 6 & 0.000281 & $4.462548 \times 10^{-5}$ & 7 & $2.324176 \times 10^{-64}$ & $1.205364 \times 10^{-1,190}$ \\
10 & 6 & 0.000000 & $1.231259 \times 10^{-7}$ & 7 & $1.392265 \times 10^{-124}$ & $1.840079 \times 10^{-2,580}$ \\
30 & 5 & 0.000000 & $2.416285 \times 10^{-34}$ & 5 & $2.416285 \times 10^{-34}$ & $1.294365 \times 10^{-1,987}$ \\
\hline
\end{tabular}

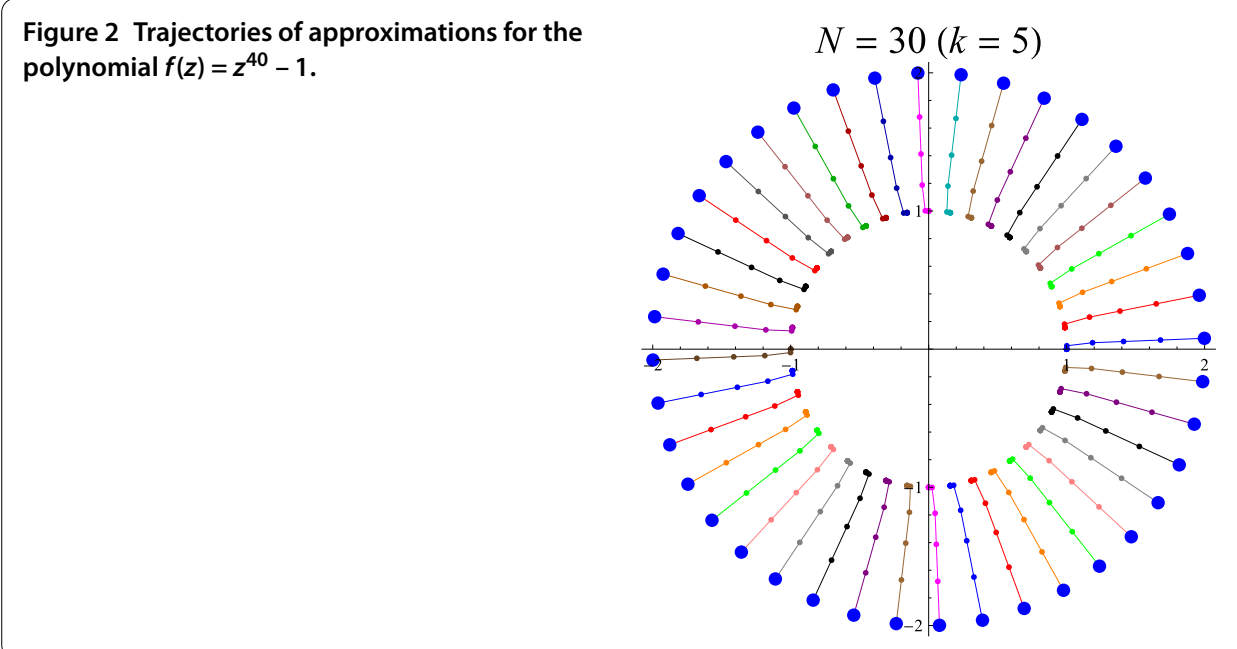

In this example we use Aberth's initial approximation (7.7) with $a_{1}=0, r_{0}=2$, and $n=40$. We have $\mathscr{R}=0.018685, E\left(x^{(0)}\right)=0.159318$. The results for Example 7.4 can be seen in Table 5 .

In Figure 2, we present the trajectories of approximations generated by the method (1.18) for $N=30$ after 5 iterations.

Competing interests

The authors declare that they have no competing interests.

Authors' contributions

Both authors contributed equally and significantly in writing this paper. Both authors read and approved the final manuscript.

\section{Acknowledgements}

This research is supported by the project NI15-FMI-004 of Plovdiv University.

Received: 14 August 2015 Accepted: 5 October 2015 Published online: 19 October 2015

\section{References}

1. Proinov, PD: A unified theory of cone metric spaces and its applications to the fixed point theory. Fixed Point Theory Appl. 2013, Article ID 103 (2013)

2. Sendov, B, Andreev, A, Kjurkchiev, N: Numerical Solution of Polynomial Equations. In: Handbook of Numerical Analysis, vol. III, pp. 625-778. Elsevier, Amsterdam (1994)

3. Kyurkchiev, NV: Initial Approximations and Root Finding Methods. Mathematical Research, vol. 104. Wiley, Berlin (1998)

4. McNamee, JM: Numerical Methods for Roots of Polynomials - Part I. Studies in Computational Mathematics, vol. 14. Elsevier, Amsterdam (2007) 
5. Petković, M: Point Estimation of Root Finding Methods. Lecture Notes in Mathematics, vol. 1933. Springer, Berlin (2008)

6. Weierstrass, K: Neuer Beweis des Satzes, dass jede ganze rationale Function einer Veränderlichen dargestellt werden kann als ein Product aus linearen Functionen derselben Veränderlichen. Sitzungsber. Königl. Preuss. Akad. Wiss. Berlin II, 1085-1101 (1891)

7. Ehrlich, LW: A modified Newton method for polynomials. Commun. ACM 10(2), 107-108 (1967)

8. Aberth, O: Iteration methods for finding all zeros of a polynomial simultaneously. Math. Comput. 27, 339-344 (1973)

9. Börsch-Supan, W: Residuenabschatzung für Polynom-Nullstellen mittels Lagrange-Interpolation. Numer. Math. 14, 287-296 (1970)

10. Werner, W: On the simultaneous determination of polynomial roots. In: Iterative Solution of Nonlinear Systems of Equations. Lecture Notes Math., vol. 953, pp. 188-202. Springer, Berlin (1982)

11. Proinov, PD: On the local convergence of the Ehrlich method for numerical computation of polynomial zeros. Calcolo (2015). doi:10.1007/s10092-015-0155-y

12. Kyurkchiev, NV, Taschev, S: A method for simultaneous determination of all roots of algebraic polynomials. C. R. Acad. Bulg. Sci. 34, 1053-1055 (1981) (in Russian)

13. Tashev, S, Kyurkchiev, N: Certain modifications of Newton's method for the approximate solution of algebraic equations. Serdica Math. J. 9, 67-73 (1983) (in Russian)

14. Wang, DR, Zhao, FG: Complexity analysis of a process for simultaneously obtaining all zeros of polynomials. Computing 43, 187-197 (1989)

15. Tilli, P: Convergence conditions of some methods for the simultaneous computation of polynomial zeros. Calcolo 35 3-15 (1998)

16. Kjurkchiev, NV, Andreev, A: Ehrlich's methods with a raised speed of convergence. Serdica Math. J. 13, $52-57$ (1987)

17. Proinov, PD: General local convergence theory for a class of iterative processes and its applications to Newton's method. J. Complex. 25, 38-62 (2009)

18. Proinov, PD: New general convergence theory for iterative processes and its applications to Newton-Kantorovich type theorems. J. Complex. 26, 3-42 (2010)

19. Proinov, PD: General convergence theorems for iterative processes and applications to the Weierstrass root-finding method. J. Complex. (2015, accepted). arXiv:1503.05243

20. Proinov, PD, Cholakov, SI: Semilocal convergence of Chebyshev-like root-finding method for simultaneous approximation of polynomial zeros. Appl. Math. Comput. 236, 669-682 (2014)

21. Proinov, PD, Vasileva, MT: On the convergence of a family of Weierstrass-type root-finding methods. C. R. Acad. Bulg. Sci. 68, 697-704 (2015)

22. Proinov, PD: Relationships between different types of initial conditions for simultaneous root finding methods. Appl. Math. Lett. 52, 102-111 (2016)

23. Wolfram Mathematica, version 7. Wolfram Research, Inc., Champaign, Illinois, USA (2009)

24. Zhang, X, Peng, H, Hu, G: A high order iteration formula for the simultaneous inclusion of polynomial zeros. Appl. Math. Comput. 179, 545-552 (2006)

25. Petković, M, Ilić, S, Petković, I: A posteriori error bound methods for the inclusion of polynomial zeros. J. Comput. Appl. Math. 208, 316-330 (2007)

26. Wilkinson, JH: Rounding Errors in Algebraic Processes. Prentice Hall, New York (1963)

\section{Submit your manuscript to a SpringerOpen ${ }^{\circ}$ journal and benefit from:}

- Convenient online submission

Rigorous peer review

- Immediate publication on acceptance

- Open access: articles freely available online

- High visibility within the field

- Retaining the copyright to your article 